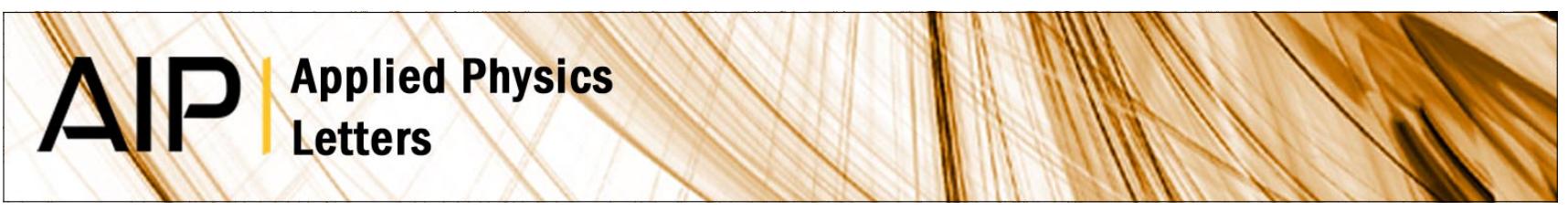

\title{
Thickness dependence of the integrated Bragg intensity for statistically disturbed silicon crystals
}

J. Will, A. Gröschel, M. Weißer, and A. Magerl

Citation: Appl. Phys. Lett. 98, 041910 (2011); doi: 10.1063/1.3531761

View online: http://dx.doi.org/10.1063/1.3531761

View Table of Contents: http://apl.aip.org/resource/1/APPLAB/v98/i4

Published by the American Institute of Physics.

\section{Related Articles}

A reaction diffusion model of pattern formation in clustering of adatoms on silicon surfaces AlP Advances 2, 042101 (2012)

Convergent beam electron-diffraction investigation of lattice mismatch and static disorder in GaAs/GaAs1-xNx intercalated GaAs/GaAs1-xNx:H heterostructures

Appl. Phys. Lett. 101, 111912 (2012)

Response to "Comment on "Elastic wave propagation in a solid layer with laser-induced point defects"' [J. Appl.

Phys. 112, 056101 (2012)]

J. Appl. Phys. 112, 056102 (2012)

Comment on "Elastic wave propagation in a solid layer with laser-induced point defects" [J. Appl. Phys. 110, 064906 (2011)]

J. Appl. Phys. 112, 056101 (2012)

Response to "Comment on 'Intrinsic point defect incorporation in silicon single crystals grown from a melt, revisited'" [J. Appl. Phys. 111, 116102 (2012)]

J. Appl. Phys. 111, 116103 (2012)

\section{Additional information on Appl. Phys. Lett.}

Journal Homepage: http://apl.aip.org/

Journal Information: http://apl.aip.org/about/about_the_journal

Top downloads: http://apl.aip.org/features/most_downloaded

Information for Authors: http://apl.aip.org/authors

\section{ADVERTISEMENT}
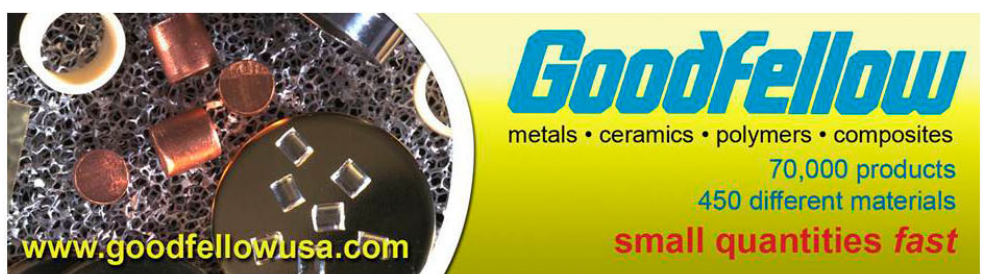


\title{
Thickness dependence of the integrated Bragg intensity for statistically disturbed silicon crystals
}

\author{
J. Will, ${ }^{\text {a) }}$ A. Gröschel, M. Weißer, and A. Magerl \\ Crystallographie and Structural Physics, University of Erlangen-Nürnberg, Staudtstr. 3, \\ 91058 Erlangen, Germany
}

(Received 9 August 2010; accepted 6 December 2010; published online 28 January 2011)

\begin{abstract}
The thickness dependence of the integrated Bragg intensities for Czochralski-grown silicon was measured with the characteristic tungsten $K_{\alpha 1}$-line at $59.3 \mathrm{keV}$. In contrast to previous experiments the sample is wedge shaped, which allows to take data over a wide range of Pendellösung fringes in one exposure only and without any mechanical movement of the sample. The period length, the oscillation amplitude, and the mean value of the Bragg intensity can be explored to identify the presence of point defects, and the temperature dependence of the period length allows to quantify the thermal Debye-coefficient with high precision. () 2011 American Institute of Physics.

[doi:10.1063/1.3531761]
\end{abstract}

The presence of statistically distributed point defects such as electric dopants or oxygen in Czochralski-grown silicon leads to a distortion of the crystal lattice planes. The resulting strain can be detected with high sensitivity in an $\mathrm{x}$-ray measurement via the behavior of the Pendellösung oscillations. The dynamical diffraction of statistically disturbed crystals was originally developed by Kato ${ }^{1}$ and modified later by $\mathrm{Al} \mathrm{Haddad}$ and Becker. ${ }^{2}$ In the following, we first present a setup at our High Energy x-ray Laboratory, Bayern, Bavaria, to measure Pendellösung oscillations. Subsequently, we present experimental results for Czochralski-grown silicon at room temperature and up to $450{ }^{\circ} \mathrm{C}$. The data are compared to the results within the framework of the theory for a statistically disturbed crystal, and they are evaluated in view of the thermal Debye-coefficient.

A tungsten tube with a $0.6 \times 0.6 \mathrm{~mm}^{2}$ focal spot and a maximum acceleration voltage of $225 \mathrm{kV}$ has been used as an x-ray source. The measurements were taken at the characteristic tungsten $K_{\alpha 1}$-line at $59.3 \mathrm{keV}$ in a focusing Laue setup. ${ }^{3}$ Two samples had been prepared from a Czochralskigrown silicon crystal. These were cut from a $15 \mathrm{~mm}$ thick and 6 inch diameter silicon rod with a diamond saw into a wedge-shaped geometry. The wedge angle was chosen big enough to measure for the $\operatorname{Si}\langle 400\rangle$-reflection at least one dozen of Pendellösung oscillations with only one exposure. At the same time the wedge angle should be small enough such that the oscillation amplitude is not significantly smeared out by the finite size of the X-ray source. A beam divergence of $0.8^{\circ}$ and a distance from source to sample of $3 \mathrm{~m}$ result in a beam height of about $4 \mathrm{~cm}$, and a wedge angle of $3^{\circ}$ appears appropriate (see Fig. 1). The samples were etched with a mixture of hydrofluoric and nitric acid to remove surface strain from the sawing process. The thickness of both samples at the tip of the wedge is about $70 \mu \mathrm{m}$. The samples have $8 \times 10^{17}$ oxygen atoms per $\mathrm{cm}^{3}$ and they are further doped with $2 \times 10^{15}$ boron atoms per $\mathrm{cm}^{3}$.

Figure 2 compares the experimental data for the 400 reflection at room temperature to the theory of an absorbing ideal crystal characterized by a Pendellösung distance of $152 \mu \mathrm{m},{ }^{4}$ which includes a room temperature Debyecoefficient $B$ of $0.4882 .{ }^{5}$ The intensity axis has been adjusted

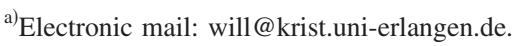

between the positions of the first maxima and minima as the only adjustable parameter. Obviously, there are deviations in the periodicity, in the oscillation amplitude, and in the progression of the integrated intensity. These discrepancies are explained coherently by Kato's statistical dynamical theory. Two significant parameters $E$ and $\tau$ have been introduced by Kato, where $E$ is a long range order parameter, which is equal to 1 for an ideal perfect crystal. Otherwise it becomes smaller than 1 and approaching 0 for an ideal imperfect crystal. The deviation from the value 1 depends on the observed lattice reflection $h k l$, and it can be interpreted as a lost volume for the coherent diffracted waves due to disturbances of the crystal. $E$ can be approximated as $E(h, k, l) \approx 1$ $-\Delta V(h, k, l) / V$, where $V$ is the total volume of the sample and $\Delta V(h, k, l)$ is associated with the disturbed volume fraction, also denominated incoherent volume. $E$ is also referred to as the static Debye-Waller-factor, as it denotes the loss of lattice phase coherence due to static disorder. $\tau$ is a short range order parameter representing the average correlation length of the lattice distortions.

Figure 3 compares the experimental data from Fig. 2 with the statistical dynamical theory ${ }^{1,2}$ (solid line) with values for $E$ and $\tau$ of 0.98 and $180 \mathrm{~nm}$, respectively. The resulting elongation of the Pendellösung distance by $0.12 \%$ together with the simultaneous diminution of the oscillation

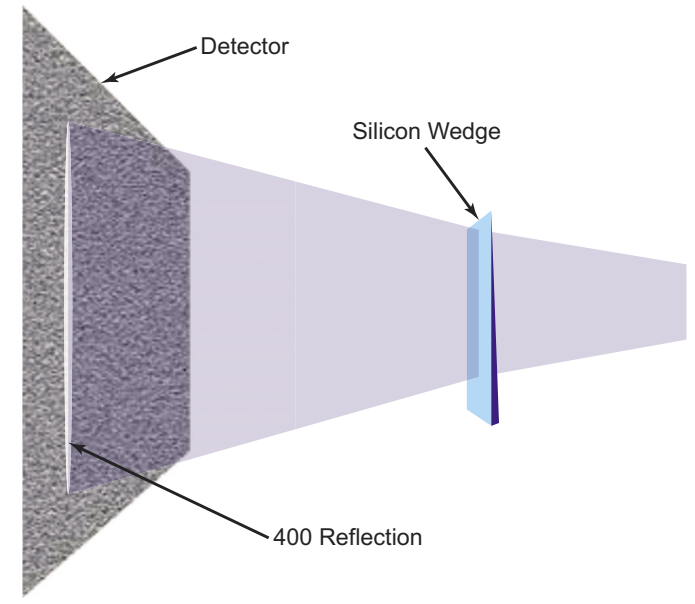

FIG. 1. (Color online) Focusing Laue-geometry for a wedge-shaped crystal. 


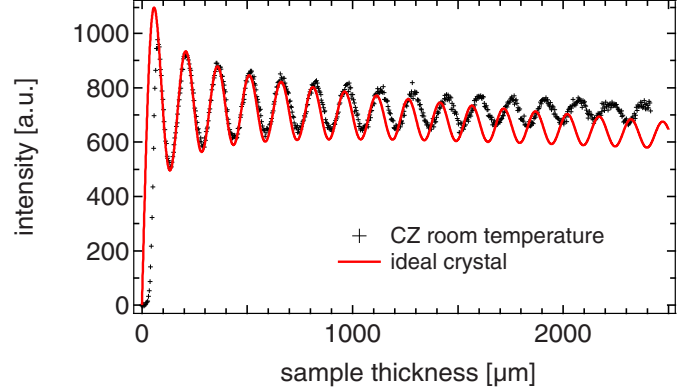

FIG. 2. (Color online) 400 Pendellösung oscillations of a Czochralskigrown silicon crystal at room temperature (crosses) measured at $59.3 \mathrm{keV}$ compared to the ideal crystal behavior (solid line) with a period length of $152 \mu \mathrm{m}$ (Ref. 4).

amplitude and the increasing mean value of the Bragg intensity with the crystal thickness describe the measurement well. We argue that the identified strain inventory originates from the dopings and mainly from oxygen, which has by far the highest concentration. In further experiments we could assure that strain from the sample preparation including the sawing is irrelevant.

It appears that measurements of wedge-shaped silicon samples at room temperature are an easy, however, extremely sensitive means to identify the presence of point defects in the crystal lattice. Further studies, in particular, on a float-zone material will be conducted for a full quantitative assessment of strain fields in doped silicon and other high quality crystals of relevance for applications in semiconductor and optical industries.

The thermal vibrational amplitudes of the atoms vary with temperature as usually expressed by the thermal Debye-Waller-factor. This leads to a reduction in the structure factor, and in consequence to an elongation of the Pendellösung distance. ${ }^{6}$ Thus, the total elongation of the Pendellösung distance will in general be the product of the thermal Debye-Waller-factor $M$ and the static DebyeWaller-factor $E$.

The measured data show the thickness dependent intensity oscillations together with the thickness dependence of the mean value of the Bragg intensity. We note that an increase in $M$ will elongate the periodicity and cause in addition a decrease in the mean value of the intensity, which should be more pronounced for higher order reflections. In contrast, the presence of a strain will change both $E$ and $\tau$ resulting in an elongation of the periodicity and simultaneously giving rise to an intensity progression with sample thickness. These different fingerprints provide a sensitive

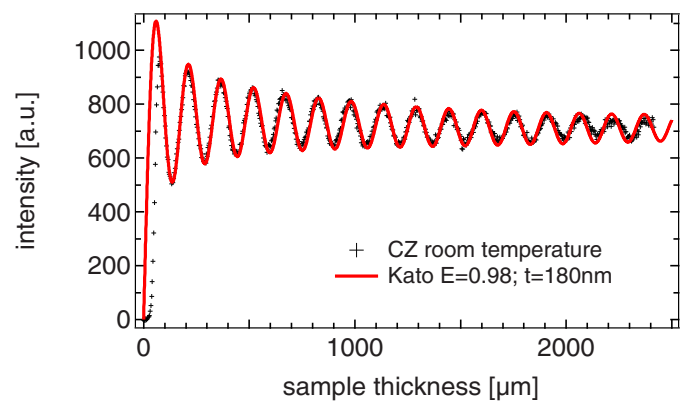

FIG. 3. (Color online) Measurement of a Czochralski-grown silicon crystal compared to the theory of a statistically disturbed crystal with $E=0.98$ and $\tau=180 \mathrm{~nm}$.

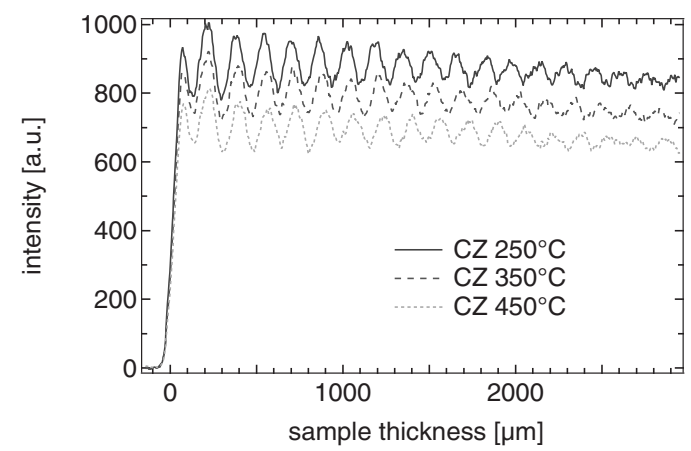

FIG. 4. Elongation of the Pendellösung distance with temperature: (solid gray line) $250{ }^{\circ} \mathrm{C}$, (dashed light gray line) $350{ }^{\circ} \mathrm{C}$, and (black dotted line) $450{ }^{\circ} \mathrm{C}$. The data are shifted vertically for increased clarity.

means to separate thermal motions and static strain.

Recent temperature dependent measurements in pink beam Laue transmission geometry on the strain inventory in Czochralski-grown silicon crystals show that a rearrangement of oxygen interstitials with the formation of precipitates sets in at temperatures above $800{ }^{\circ} \mathrm{C}$, while the integrated intensity is constant below this temperature. ${ }^{7}$

Figure 4 compares at 250,350 , and $450{ }^{\circ} \mathrm{C}$, a regime where it is usually assumed that oxygen mobility can be neglected for the build-up of strain. The data clearly show a variation of the oscillation period with temperature. Further careful inspection reveals a slight reduction in the mean value of the Bragg intensity with rising temperature. Thus, the increase in the periodicity can be related to a decreasing structure factor originating from an increasing thermal Debye-coefficient, while the strain inventory remains constant in a temperature range up to $450{ }^{\circ} \mathrm{C}$.

In Table I the Pendellösung distances measured at high temperatures are compared to the room temperature values (see Fig. 3). In all cases a static Debye-Waller-factor $E$ of 0.98 is assumed, which is justified because all samples were cut from the same bulk material. The analysis of the measured Pendellösung distances leads to a temperature dependent Debye-coefficient based on the room temperature Debye-coefficient of $0.4882 .^{5}$ Here the thermal DebyeWaller-factor is $\exp \left(-B s^{2}\right)$, with $s=\sin (\theta) / \lambda$, and $\theta$ and $\lambda$ denote the scattering angle and the $\mathrm{x}$-ray wavelength, respectively.

In conclusion, the experimental setup presented is a simple, but highly sensitive means to identify via Pendellösung oscillations strain from point defects or small precipitates in high quality single crystals and to measure the thermal Debye-Waller-factor in a wide temperature range with outstanding precision. Both effects can be uniquely identified from the correlated changes in the period length, the oscilla-

TABLE I. Measured data of the Pendellösung distance $\Lambda_{400}$ as a function of temperature. For comparison the Pendellösung distance at room temperature is also given [see Fig. $3(E=0.98)$ ]. $F_{400}$ is the 400 structure factor calculated out of $\Lambda_{400}$ and $B$ the Debye-coefficient.

\begin{tabular}{lrrrc}
\hline \hline Temperature & $250{ }^{\circ} \mathrm{C}$ & $350{ }^{\circ} \mathrm{C}$ & $450{ }^{\circ} \mathrm{C}$ & $22{ }^{\circ} \mathrm{C}$ \\
\hline$\Lambda_{400}(\mu \mathrm{m})$ & 160.3 & 163.6 & 166.6 & 154.9 \\
$F_{400}(T) / F_{400}\left(22{ }^{\circ} \mathrm{C}\right)$ & 0.966 & 0.947 & 0.930 & 1 \\
$B$ & 0.743 & 0.889 & 1.023 & $0.4882^{\mathrm{a}}$ \\
\hline \hline
\end{tabular}

${ }^{\mathrm{a}}$ Reference 5 . 
tion amplitude, and the mean value of the intensity. The data can still be improved further by evaluating simultaneously different Bragg reflections.

We acknowledge the support by $\mathrm{H}$. Grillenberger for making the Si material available.

${ }^{1}$ N. Kato, Acta Crystallogr., Sect. A: Cryst. Phys., Diffr., Theor. Gen. Crystallogr. 36, 763 (1980)
${ }^{2}$ M. Al Haddad and P. J. Becker, Acta Crystallogr., Sect. A: Found. Crystallogr. 44, 262 (1988).

${ }^{3}$ M. Stockmeier and A. Magerl, J. Appl. Crystallogr. 41, 754 (2008).

${ }^{4}$ S. Stepanov, see: http://sergey.gmca.aps.anl.gov/x0h.html.

${ }^{5}$ L.-M. Peng, G. Ren, L. Dudarev, and M. J. Whelan, Acta Crystallogr., Sect. A: Found. Crystallogr. 52, 456 (1996).

${ }^{6}$ A. Authier, Dynamical Theory of X-Ray Diffraction (Oxford University Press, Oxford, 2001).

${ }^{7}$ H. Grillenberger and M. Magerl, J. Appl. Phys. 106, 024910 (2009). 